\title{
On the interrelation between ionization of light atoms by virtual and real photons
}

\author{
A.B.Voitkiv and J.Ullrich \\ Max-Planck-Institut für Kernphysik, Saupfercheckweg 1, D-69117, Heidelberg, Germany \\ (J.Phys.B, accepted)
}

\begin{abstract}
The correspondence between ionization of light atoms by charged particle impact (virtual photons) and by photoabsorption (real photons) is analysed. It is shown that the "genuine" photo-like mechanism in the impact ionization of light targets can be already observed at collision energies corresponding to the Lorentz factor $\gamma \gtrsim 10$, but that it starts to dominate electron emission only at $\gamma \gtrsim 1000$. It is demonstrated that the nonrelativistic Bethe-Born and Weizsäcker-Williams approximations represent extremely different limits of the impact ionization but that "by chance" their results are formally close for electron emission in nonrelativistic fast collisions.
\end{abstract}

PACS:34.10.+x, 32.80.-t, 32.80.Fb 
The relationship between ionization of light atoms by charged projectiles and photons is of fundamental importance for detailed understanding of the atomic response to the action of electromagnetic fields. Assuming fast but nonrelativistic collision velocities, this relationship has been extensively discussed in the atomic physics literature during the last two decades (see e.g. [1]- [3] and reference therein). Within the nonrelativistic Bethe-Born (NBB) dipole limit, atom ionization by a projectile in collisions with small momentum transfer is often considered by stressing certain similarities with atom ionization due to photoabsorption (photoeffect). Cross sections for atom ionization in such collisions can be expressed via photo cross sections.

The Weizsäcker-Williams (WW) method of equivalent photons [4], which is widely used in nuclear and high-energy physics [5], has recently become an ordinary tool to estimate electron emission produced in collisions of light atoms with energetic heavy projectiles (see e.g. [6], [7]). This method is based on the fact that in collisions with not too small impact parameters the field of a (ultra) relativistic projectile can be nearly equivalent to pulses of a (free) radiation field. As a result ionization cross sections in collisions with charged projectiles can be directly related to photoabsorption cross sections. Taking into account the relativistic "origin" of the WW method, one usually routinely states that this method can only be applied for collision energies larger than a few $\mathrm{GeV} / \mathrm{n}$ (see e.g. [6] ). However, it is sometimes used even for nonrelativistic fast collisions with light targets [8], [6] where it still seems to be able to yield reasonable results for ionization cross sections.

Having in mind all this, some questions appear. What are the "genuine" validity limits of the WW method to treat different aspects of ionization of light targets in collisions with charged particles? Is the "practical" success of the WW method in describing electron emission in fast nonrelativistic collisions entirely accidental or there still are some physical reasons for it? Is there some correspondence between the NBB and WW approximations? If both seem to reproduce reasonably well atomic cross sections in nonrelativistic fast collisions, then does it mean that they imply similar ionization mechanisms in this range? And how the ionization mechanisms in the NBB and WW approximations are related to the mechanism of the photoeffect?

Althougt the NBB and WW approximations have been used in many papers to calculate cross sections for light atoms and there is a number of books and reviews on atomic collisions (see e.g. [1]- [3], [5], [9]- [11]), where these approximations are discussed, we were not able to locate in the literature clear answers on the points stated above. The present communication addresses these points by considering the interrelation between single ionization of a light atom by a collision with a fast charged projectile and due to photoeffect. Since the electromagnetic field is essentially a relativistic object, a real insight into the interrelation can only be obtained if one treats the field of a projectile relativistically.

Let us consider the collision between a light atom and a point-like particle with a charge $Z_{p}$. For the sake of simplicity the atom is assumed to be a single-electron system. As a reference frame we take the frame where initially the atomic target is at rest and the particle-projectile moves with a velocity $\mathbf{v}$. The collision will be treated within the first order in the projectile-target interaction. Since it is convenient to consider the atomic photo effect by employing the Coulomb gauge, we will use this gauge throughout the paper.

As a result of the projectile-target collision a momentum $q$ is transferred to the atom and the latter makes a transition from its initial state $\psi_{0}$ with an energy $\varepsilon_{0}$ to a final continuum 
state $\psi_{\mathbf{p}}$ with momentum $\mathbf{p}$ and energy $\varepsilon_{\mathbf{p}}$. The cross section for the process, differential in the momentum transfer $\mathbf{q}$, reads (see e.g. [12])

$$
\frac{d \sigma_{0 \rightarrow \mathbf{p}}}{d \mathbf{p} d \mathbf{q}_{\perp}}=\frac{4 Z_{p}^{2}}{v^{2}}\left|\left\langle\psi_{\mathbf{p}}(\mathbf{r})|\hat{T}| \psi_{0}(\mathbf{r})\right\rangle\right|^{2},
$$

where the operator $\hat{T}$ is given by

$$
\hat{T}=\frac{\exp (i \mathbf{q} \cdot \mathbf{r})}{\mathbf{q}_{\perp}^{2}+q_{\min }^{2}}-\frac{c \exp (i \mathbf{q} \cdot \mathbf{r}) \mathbf{g} \cdot \boldsymbol{\alpha}}{\mathbf{q}_{\perp}^{2}+\frac{q_{\min }^{2}}{\gamma^{2}}} .
$$

In Eqs.(1)-(2) $\mathbf{r}$ are the electron coordinates and $\mathbf{q}=\left(\mathbf{q}_{\perp}, q_{\min }\right)$ is the momentum transfer, where $\mathbf{q}_{\perp}$ is its two-dimensional part which is perpendicular to the projectile velocity $\mathbf{v}$ and $q_{\min }=\frac{\varepsilon_{\mathbf{p}}-\varepsilon_{0}}{v}$ is the momentum component in the direction of $\mathbf{v}$. Further, $\mathbf{g}=\frac{1}{c^{2}}\left(\mathbf{v}-\frac{\varepsilon_{\mathbf{p}}-\varepsilon_{0}}{q^{2}} \mathbf{q}\right), c=137$ a.u. is the speed of light, $\gamma=1 / \sqrt{1-v^{2} / c^{2}}$, and $\boldsymbol{\alpha}$ are the Dirac matrices for the atomic electron. We note that $\mathbf{g} \cdot \mathbf{q}=0$.

In Eq.(1) the initial and final electron states are assumed to be relativistic fourcomponent wavefunctions. It is known, however, that even for relativistic collisions only a vanishingly small part of electrons emitted from light targets can attain relativistic energies in the target frame. Since nonrelativistic wavefunctions are in general much easier to handle, one can try to transform the cross section (1) in a way which allows one to use the nonrelativistic description for the initial and final electron states. This can be done by expressing in (1) the "small" components of $\psi_{0}$ and $\psi_{\mathbf{p}}$ via their "large" components and neglecting the term, which contains the product of the two "small" componets. If one also neglects the spin-flip terms, which are known to be of negligible importance for ionization of light targets [13], [14], then the result is

$$
\frac{d \sigma_{0 \rightarrow \mathbf{p}}}{d \mathbf{p} d \mathbf{q}_{\perp}}=\frac{4 Z_{p}^{2}}{v^{2}}\left|\left\langle\phi_{\mathbf{p}}(\mathbf{r})\left|\hat{T}_{1}\right| \phi_{0}(\mathbf{r})\right\rangle\right|^{2} .
$$

In Eq.(3) $\phi_{0}$ and $\phi_{\mathbf{p}}$ are the nonrelativistic wavefunctions, which are solutions of the Schrödinger equation for a free target. The operator $\hat{T}_{1}$ is given by

$$
\hat{T}_{1}=\frac{\exp (i \mathbf{q} \cdot \mathbf{r})}{\mathbf{q}_{\perp}^{2}+q_{\min }^{2}}-\frac{\exp (i \mathbf{q} \cdot \mathbf{r}) \mathbf{g} \cdot \hat{\mathbf{p}}}{\mathbf{q}_{\perp}^{2}+\frac{q_{\min }^{2}}{\gamma^{2}}} .
$$

As a check, we used (3) to calculate electron spectra emitted from light hydrogen-like ions in collisions with projectiles for a broad range of collision energies corresponding to $1<\gamma \leq 10^{6}$ and found that (3) represents an excellent approximation to (1). ${ }^{1}$

\footnotetext{
${ }^{1}$ Eqs.(3)-(4) can also be obtained from the treatment of [15]. The authors of [15] used the classical impact parameter description for the projectile motion and considered the Dirac equation for the target electron moving in the field of the target nucleus and of the projectile. By means of analysis of the structure of the Dirac equation a modified Schrödinger equation, where the terms quadratic in the projectile potentials are of minor importance and can be omitted, was proposed to treat excitation and ionization of light targets in collisions with relativistic projectiles. A similar form of the modified Schrödinger equation was suggested very recently [16] after analysing the KleinGordon equation.
} 
The transition matrix element for photo ionization of a light single-electron target is proportional to

$$
\left\langle\phi_{\mathbf{p}}|\exp (i \mathbf{k} \cdot \mathbf{r})(\mathbf{e} \cdot \hat{\mathbf{p}})| \phi_{0}\right\rangle
$$

where $\mathbf{e}$ is the photon polarization and $\mathbf{k}$ the photon momentum. For a real photon one has $\mathbf{k} \cdot \mathbf{e}=0$ and $\sum_{\mu=0}^{3} k_{\mu} k^{\mu}=0$. For $k a_{0} \ll 1$, where $a_{0}$ is the typical target size, setting $\exp (i \mathbf{k} \cdot \mathbf{r})=1$ and taking into account the identity $\left\langle\phi_{\mathbf{p}}|\hat{\mathbf{p}}| \phi_{0}\right\rangle=i \omega\left\langle\phi_{\mathbf{p}}|\mathbf{r}| \phi_{0}\right\rangle$, where $\omega=\varepsilon_{\mathbf{p}}-\varepsilon_{0},(5)$ reduces to

$$
i \omega\left\langle\phi_{\mathbf{p}}|\mathbf{e} \cdot \mathbf{r}| \phi_{0}\right\rangle
$$

According to (3) and (4) the transition matrix element for collisional ionization is proportional to

$$
\frac{\left\langle\phi_{\mathbf{p}}|\exp (i \mathbf{q} \cdot \mathbf{r})| \phi_{0}\right\rangle}{q^{2}}-\frac{\left\langle\phi_{\mathbf{p}}|\exp (i \mathbf{q} \cdot \mathbf{r})(\mathbf{g} \cdot \hat{\mathbf{p}})| \phi_{0}\right\rangle}{q^{2}-\frac{\omega^{2}}{c^{2}}} .
$$

In this expression $\mathbf{q}=\left(\mathbf{q}_{\perp}, q_{\text {min }}\right)$ and $\omega=\varepsilon_{\mathbf{p}}-\varepsilon_{0}$ are now regarded as the momentum and energy of a virtual photon which represents the projectile field. There are two different types of transitions which are given by the first and second terms in (7). The first type is due to the interaction with a so called longitudinal virtual photon. The exchange of such a photon exerts a force which is parallel to the momentum transfer $\mathbf{q}$. The other type of force is transmitted by the exchange of a transverse virtual photon with the polarization vector proportional to $\mathbf{g}=\left(\mathbf{v}-\frac{\omega}{q^{2}} \mathbf{q}\right) / c^{2}$. Since this polarization vector is perpendicular to the photon momentum, $\mathbf{g} \cdot \mathbf{q}=0$, in the case of small momentum transfers, $q a_{0} \ll 1$, the second type of the force acts perpendicularly to the momentum transfer.

In high-velocity collisions ionization due to small momentum transfers, $q a_{0}<1$, is known to dominate in electron emission from light targets. For such collisions one can expand the exponentials in (7) and keep there only the first nonvanishing terms. Performing such an expansion and using $\left\langle\phi_{\mathbf{p}}|\hat{\mathbf{p}}| \phi_{0}\right\rangle=i \omega\left\langle\phi_{\mathbf{p}}|\mathbf{r}| \phi_{0}\right\rangle$, one obtains that the transition matrix element is proportional to

$$
i \omega\left\langle\phi_{\mathbf{p}}|\mathbf{G} \cdot \mathbf{r}| \phi_{0}\right\rangle
$$

where

$$
\mathbf{G}=\frac{\mathbf{q}}{\omega q^{2}}+\frac{1}{\omega^{2}-c^{2} q^{2}}\left(\mathbf{v}-\frac{\omega}{q^{2}} \mathbf{q}\right) .
$$

The vector $\mathbf{G}$ corresponds to the vector $\mathbf{e}$ in the photoionization (see (6)) and is proportional to the polarization of a virtual photon which represents the projectile field. This photon can be thought of as a "compound" virtual photon which is constituted of the longitudinal and transverse components. According to (8), the vector $\mathbf{G}$ forms a symmetry axis for electrons emitted in collisions with small momentum transfers. By means of simple manipulations of Eq.(9) one can show that this axis forms an angle

$$
\theta_{\mathbf{G}}=\arctan \left(\frac{\gamma^{2} q_{\perp}}{\omega / v}\right)
$$


with the projectile velocity $\mathbf{v}$ and in general does not coincide with the direction of the momentum transfer $\mathbf{q}$. This is in contrast to the NBB limit which yields simply

$$
\frac{i}{q^{2}}\left\langle\phi_{\mathbf{p}}|\mathbf{q} \cdot \mathbf{r}| \phi_{0}\right\rangle
$$

and predicts the electron emission to be symmetric along the momentum transfer q. Thus, the relativistic effects tend to shift the symmetry axis towards larger emission angles and this shift can be rather substantial even for moderate values of $\gamma$ as illustrated in figure 1 .

One can expect the processes of photo and impact ionization to be very closely interrelated if the latter ionization is caused by the exchange of a "compound" virtual photon with a "strong" transverse and a "weak" longitudinal component, especially if the energymomentum properties of such a virtual photon would also be very close to those of a real photon. Comparing the contributions of the longitudinal and transverse components to the electron transition matrix element (8) one can show that the transverse component of a "compound" photon dominates the transitions if

$$
\frac{\omega}{v \gamma^{2}} \ll q_{\perp} \ll \frac{\omega}{v} \quad\left(\frac{q_{\min }}{\gamma^{2}} \ll q_{\perp} \ll q_{m i n}\right) .
$$

Note that (12) can become true only if $\gamma \gg 1$. A virtual photon, whose four-momentum components satisfy the above conditions, has properties which are already very close to those of a real photon: i) it is almost transversely polarized, $\mathbf{G} \cdot \mathbf{q} \approx 0(\mathbf{G} \cdot \mathbf{q} /(G q) \ll 1)$, and ii) one has $q_{\nu} q^{\nu} \approx 0$ on the relevant energy-momentum scale $\left(q_{\nu} q^{\nu}=q_{\perp}^{2}+\frac{\omega^{2}}{v^{2} \gamma^{2}} \ll(\omega / c)^{2}\right)$, i.e. the virtual photon is almost on mass shell. In this case emission pattern of electrons due to charged particle impact (an example of such a pattern is given by dot curve in figure 1) is nearly identical to that due to the photo effect. Thus, an exchanged photon is "quasi-real" and the mechanism of ionization by a charged projectile is quite photo-like provided (12) is fulfilled.

By analysing the two terms in (7) one can convince oneself that collisions with larger values of the momentum transfer, $q \gg \omega / v$, are always dominated by the exchange of a longitudinal photon. In addition, the exchange of a longitudinal photon always dominates collisions where $q_{\perp}$ is very small, $q_{\perp} \ll \frac{\omega}{v \gamma^{2}}$. The latter condition leads to important conceptual result that collisions with minimum momentum transfer, $q=q_{\text {min }}$, which are often termed as the optical limit and regarded to be closest to the photoabsorption [3], in fact can never be photo-like.

Thus, the fundamental similarity between ionization of a light atomic system due to photoeffect and charged particle impact can be observed only in the domain of ultrarelativistic collision energies, $\gamma \gtrsim 10$, and even within this domain the photo-like ionization mechanism dominates the target ionization only for that part of projectile-target collisions which satisfy the conditions (12).

In the semi-classical impact-parameter description of electronic emission, taking into account that collisional impact parameter $\mathbf{b}$ is conjugate, in the sense of the Fourier transform, to $\mathbf{q}_{\perp}$ and $b \sim 1 / q_{\perp}$, the conditions (12) can be reformulated as

$$
\frac{v}{\omega} \ll b \ll \frac{v \gamma^{2}}{\omega} .
$$


The contribution to the emission of electrons with an energy $\varepsilon_{p}=\omega+\varepsilon_{0}$ from collisions with impact parameters $b>v \gamma / \omega$, where the effective collision time $T(b) \sim b /(\gamma v)$ is larger than the typical electron transition time $\tau \sim 1 / \omega$, is negligible because the perturbation acting on the electron in such collisions is not only very weak but also adiabatically slowly changing in time. Hence, the impact parameter range, where atom ionization in collisions with charged projectiles closely resembles that due to photoabsorption, is effectively restricted to

$$
\frac{v}{\omega} \ll b \lesssim \frac{v \gamma}{\omega} .
$$

The main contribution to soft electron emission is known to be given by the impact parameter region $a_{0} \lesssim b \lesssim v \gamma / \omega_{e f f}$, where $\omega_{\text {eff }}$ is the effective frequency for the dipole atomic transitions to the continuum. This region can be subdivided into two ranges. In the first range, roughly given by $a_{0} \lesssim b \lesssim v / \omega_{\text {eff }}$ and contributing to electron emission as $\sim \ln \left(v /\left(a_{0} \omega_{\text {eff }}\right)\right)$, the longitudinal photon exchange dominates the electron transitions. The second range, which is approximately determined by $v / \omega_{\text {eff }}<b \lesssim v \gamma / \omega_{\text {eff }}$ and whose contribution to the emission $\sim \ln \gamma$, is dominated by the exchange of transverse photons. Therefore, only at $\gamma \gg v /\left(a_{0} \omega_{e f f}\right)$ the main part of electron emission is indeed photo-like in the "genuine", above-defined sence. Since for light atomic systems, like H or He, one has $\omega_{\text {eff }} a_{0} \sim 1$ a.u., it implies that $\gamma \gg 100$. Thus, althought the "genuine" photo-like mechanism in the cross section differential in momentum transfer can be observed already starting with collision energies of $\sim 10 \mathrm{GeV} / \mathrm{n}$, it would dominate the total electron emission only at extremely high collision energies of $\gtrsim 1 \mathrm{TeV} / \mathrm{n}$. The latter energies are the true domain of the applicability of any method, which evaluates (soft) electron emission from light targets in collisions with charged particles by appealing to the fundamental analogy between the latter process and the photoeffect.

Since the WW method relies on the fundamental similarity between the projectile field and a pulse of free radiation field, its validity limits have to be the same as those found above. How, however, one can explain the "practical" success of the WW method for light targets already at $\gamma \approx 1$ ? In order to resolve this seemingly striking contradiction let us first consider the ionization mechanism in collisions at $\gamma \approx 1$. Althought the angle between the momentum $\mathbf{q}$ and the projectile velocity $\mathbf{v}$ can take any value from the interval $0 \leq \vartheta<\pi / 2$, the main contribution to electron emission in nonrelativistic fast collisions arises from the exchange of virtual photons with $q_{\perp}>\omega / v$. Such photons are essentially longitudinal photons and are polarized along q. Since $q_{\perp}$ substantially exceeds $\omega / v$ for the dominant part of ionizing collisions one has $\mathbf{q} \cdot \mathbf{v} \approx 0$ and the photons "move" almost perpendicularly to the motion of the projectile. This is in sharp contrast with the basic assumption of the equivalent photon method that the (main) pulse of the equivalent photons moves along the projectile velocity. However, the polarization of the longitudinal photons is almost transversal with respect to the projectile velocity and, therefore, is close in direction to polarization of transverse equivalent photons which, if they would exist, would move along v. Second, one can see that, in the case of light targets, the mathematical expression for the spectral density of virtual photons following from the WW method is formally similar to that of the dipole approximation ${ }^{2}$. The latter approximation, however, does not necessary

\footnotetext{
${ }^{2}$ Compare e.g. Eqs. (15.58)-(15.59) of [9] with Eq.(35) of [17], which was obtained using the
} 
imply that the collision velocity has to be relativistic. Thus, the spectral density $\frac{d I(\omega)}{d \omega}$ of the longitudinal photons is given correctly by the formulas of the WW method, where, however, the virtual photons are regarded to be nearly equivalent to real (transverse) photons. Third, a longitudinal photon does not transmit the magnetic field whereas a real photon does have both the electric and magnetic "parts". This difference, however, is not essential since the magnetic "part" of a real photon would be of minor importance for ionization of light targets. These three points explain why, despite the important conceptual difference between the longitudinal and transverse photons, the WW method can formally yield reasonable results for electron emission from light targets even at $\gamma \approx 1$. This also explains why the WW method can formally account for the contribution to the emission from the range $\omega / v \lesssim q_{\perp}<a_{0}^{-1}$ in relativistic collisions.

The physics of nonrelativistic fast collisions described by the exchange of a longitudinal photon is equivalent to that following from the NBB approximation.

In summary, in single ionization of light atomic targets by energetic projectiles the true photo-like mechanism can be observed in the cross section (1), (3) starting with $\gamma \sim 10$. The total electron emission is dominated by this mechanism at extremely high values of $\gamma$, $\gamma \gtrsim 1000$. The WW approximation, as essentially the photo-like ionization mechanism, has the same validity limits. However, because of the reasons discussed above it can formally be used to estimate electron emission even at $\gamma \sim 1$ keeping in mind that it is now a longitudinal photon which does "the job" misinterpreted as the effect of a transverse "equivalent" photon. Similar results, obtained for atomic cross section in nonrelativistic fast collisions by the application of the NBB and WW approximations, do not mean that these two may become really physically equivalent. In fact, the true validity limits of these approximations can never cross and their results can be thought of as being produced by the two distinctly different "facets", longitudinal and transverse, of a virtual photon representing the field of a fast charged projectile.

Finally we note that the same conclusions for ionization of light targets can be drawn by comparing Eqs.(1) and (2) with the relativistic form of the matrix element for the photo effect.

The authors acknowledge the support from the Deutsche Forschungsgemeinschaft (DFG) and the Leibniz-Programm of the DFG.

dipole approximation. 


\section{REFERENCES}

[1] J.H.McGuire, Electron Correlation Dynamics in Atomic Collisions (Cambridge University Press, Cambridge, England, 1997).

[2] N.Stolterfoht, R.DuBois, and R.Rivarola, Electron Emission in fast Ion-Atom Collisions (Springer-Verlag, Berlin, 1997)

[3] M.E.Rudd, Y.-K.Kim, D.H.Madison, and T.J.Gay, Rev.Mod.Phys. 64, 441 (1992)

[4] C.F.Weizsäcker, Z.Phys. 88612 (1934); E.J.Williams, Phys.Rev. 45729 (1934)

[5] C.Bertulani and G.Baur, Phys.Rep. 163299 (1988)

[6] S.Keller, H.J.Lüdde, and R.M.Dreizler, Phys.Rev. A55 4215 (1997)

[7] R.Moshammer et al, Phys.Rev.Lett. 793621 (1997)

[8] R.Moshammer et al, Phys.Rev.Lett. 771242 (1996)

[9] J.D.Jackson Classical Electrodynamics (John Wiley and Sons, 1975)

[10] M.Inokuti, Rev.Mod.Phys. 43297 (1971); Rev.Mod.Phys. 5023 (1978)

[11] J.Eichler and W.E.Meyerhof Relativistic Atomic Collisions (Academic Press, 1995)

[12] U.Fano Phys. Rev. 102385 (1956); Annu.Rev.Nucl.Sci. 131 (1963)

[13] D.M.Davidovic, B.L.Moiseiwitsch, P.H.Norrington J.Phys. B11 847 (1978)

[14] R.Anholt Phys.Rev. A 191004 (1979)

[15] P.A.Amundsen and K.Aashamar, J.Phys. B14 4047 (1981)

[16] T.Heim, K.Hencken, D.Trautmann and G.Baur, submitted to J.Phys.B; K.Heincken, private communication.

[17] A.B.Voitkiv, N.Grün and W.Scheid, J.Phys. B 323923 (1999) 


\section{FIGURE CAPTIONS}

Fig.1. Triple differential cross section (in arb. units) in the coplanar geometry for collisions of $\mathrm{H}(1 \mathrm{~s})$ with protons as a function of electron emission angle $\theta_{e m}=\arccos \left(\frac{\mathbf{p} \cdot \mathbf{v}}{p v}\right)$. $\omega=1$ a.u. and $q_{\perp}=0.001$ a.u.. Solid and dashed curves are the results of relativistic and nonrelativistic $(c \rightarrow \infty)$ calculations, respectively, for a collision energy $1 \mathrm{GeV} / \mathrm{n}(\gamma=2.07)$. Dotted curve represents the result for an ultrarelativistic collision corresponding to $\gamma=100$. Dashed arrow indicates the momentum transfer direction $\hat{\mathbf{q}}$. Solid and dotted arrows show the polarization direction $\hat{\mathbf{G}}$ of the "compound" virtual photon for $\gamma=2.07$ and $\gamma=100$, respectively. 